\title{
Controllability and hindsight components: Understanding opposite hindsight biases for self-relevant negative event outcomes
}

\author{
HARTMUT BLANK \\ University of Portsmouth, Portsmouth, England \\ AND \\ JAN H. Peters \\ University of Bamberg, Bamberg, Germany
}

\begin{abstract}
There is an anomaly in the hindsight bias literature with respect to hindsight effects obtained after self-relevant negative event outcomes: Whereas some studies have reported reduced hindsight bias, others have shown increases. This article contrasts two explanations for the anomaly. The first points to an influence of perceived control over the event outcome: In hindsight, people decrease foreseeability (and hence, responsibility and blame) for controllable events, but they increase the perceived inevitability of uncontrollable events for coping reasons. The second explanation, derived from a reconception of hindsight bias in terms of separate components (Blank, Nestler, von Collani, \& Fischer, 2008), traces the anomaly to differences in the observed hindsight components: Hindsight decreases are to be expected for foreseeability, whereas increases are restricted to the inevitability component. Our experiment $(N=210)$ manipulated controllability and the hindsight component orthogonally and showed strong support for the component explanation, but also some influence of perceived control.
\end{abstract}

For some 35 years after Fischhoff's (1975) seminal article on hindsight bias, researchers have been interested in the cognitive effects of possessing outcome knowledge (see overviews and introductions by Blank, Musch, \& Pohl, 2007; Hawkins \& Hastie, 1990; Hoffrage \& Pohl, 2003). For example, in Fischhoff's (1975) classic demonstration of hindsight bias, people who were told about the (alleged) outcome of a 19th-century military conflict between the British and the Gurkhas perceived this outcome as more likely or inevitable from the start, as compared with people without outcome knowledge. Fischhoff (1975) initially coined the term creeping determinism for this phenomenon. Other research has highlighted people's beliefs of having known the outcome all along, leading to the hindsight bias being dubbed the knew-it-all-along effect (Fischhoff, 1977). Finally, hindsight bias has been found in people's recollections of earlier predictions of events or factual outcomes (e.g., Blank, Fischer, \& Erdfelder, 2003; Fischhoff, 1977; Hell, Gigerenzer, Gauggel, Mall, \& Müller, 1988). For instance, having initially predicted that the Eiffel tower was $200 \mathrm{~m}$ tall, people received outcome feedback (the Eiffel tower is $300 \mathrm{~m}$ tall) and then, on average, remembered having made predictions of more than $200 \mathrm{~m}$ (Hell et al., 1988), thereby showing a bias toward the factual outcome.

The common denominator of all these variants of hindsight bias is that outcome knowledge biases people's hind- sight cognitions - specifically, toward perceiving events or facts to be more determined, inevitable, or foreseeable, or toward misremembering their own predictions as better than they actually were. This holds even if people are explicitly asked to ignore their knowledge about the outcome (Fischhoff, 1975). In the words of Hawkins and Hastie (1990, p. 311), "the hindsight bias is a projection of new knowledge into the past accompanied by a denial that the outcome information has influenced judgment."

Hindsight bias has long been thought to be primarily a cognitive phenomenon, a type of cognitive illusion (Pohl, 2004). Consequently, explanations of hindsight bias have focused on biased causal reasoning (e.g., Hawkins \& Hastie, 1990; Pezzo, 2003; Roese \& Olson, 1996; Wasserman, Lempert, \& Hastie, 1991) or biased memory reconstruction processes (e.g., Erdfelder \& Buchner, 1998; Pohl, Eisenhauer, \& Hardt, 2003; Stahlberg \& Maass, 1998). More recent research, however, has emphasized that self-related motivational processes play a major role in it as well (for overviews, see Musch \& Wagner, 2007; Pezzo \& Pezzo, 2007; Renner, 2003). This becomes most obvious when hindsight judgments are made regarding event outcomes that have some emotional significance for the self and invoke self-defensive processes (see Sedikides \& Gregg, 2008, for a recent overview of self-related motivational processing). It is on such self-related motiva- 
tional influence on hindsight cognitions that we focus in the present article, trying to resolve an apparent contradiction between relevant research findings.

Consider a situation in which somebody has made a decision that turns out to be a bad one. Instead of believing in hindsight that they knew this all along, as a strictly cognitive view of hindsight bias would hold (unless the outcome is surprising - in which case, hindsight bias can be attenuated or even reversed; see Müller \& Stahlberg, 2007 , for an overview), quite a number of studies have shown that hindsight is reduced and often eliminated under such conditions (Louie, 1999, 2005; Louie, Curren, \& Harich, 2000; Mark, Boburka, Eyssell, Cohen, \& Mellor, 2003; Mark \& Mellor, 1991; Pezzo \& Beckstead, 2008). Importantly, this holds even if any possibly confounding influence of surprise is controlled for (Louie, 1999). The typical explanation of reduced hindsight bias for negative decision outcomes is that people try to avoid unfavorable implications for the self that would result from showing hindsight bias in this situation. Why, if they knew all along what would happen, would they have made such a bad decision in the first place? Hence, claiming that they could not foresee the negative outcome protects them from being regarded as frivolous or outright stupid by others or, in fact, themselves (Louie, 1999, 2005; Louie et al., 2000; Mark et al., 2003; Mark \& Mellor, 1991).

So far, so good. The picture is complicated, however, by a set of studies by Tykocinski and colleagues (Tykocinski, 2001; Tykocinski, Pick, \& Kedmi, 2002; Tykocinski \& Steinberg, 2005), who drew attention to a phenomenon they termed retroactive pessimism, which can be considered a form of motivated hindsight bias. Using real-life settings, as well as scenarios, they found that people show hindsight bias especially when they are disappointed about outcomes. To illustrate, Tykocinski (2001) compared pre- and postratings of the candidates' chances in the 1999 Israeli presidential elections and found that supporters of the losing candidate were especially prone to downgrade his chances in hindsight and to inflate those of the winning candidate. Similar results, using different types of events, have been observed by Sanna and Chang (2003) and Wann, Grieve, Waddill, and Martin (2008). To explain this increased hindsight bias for personally negative outcomes, Tykocinski argued that retroactive pessimism serves to cope with disappointment: Perceiving the outcome as inevitable renders it more palatable ("I never had a chance").

The intriguing problem that arises from these two bodies of research is that both of them address hindsight consequences of self-relevant negative event outcomes and these consequences are of an opposite nature. In the decision-making case, hindsight bias is attenuated or even eliminated, and in the retroactive pessimism case, it is accentuated. How to explain this divergence? A first explanation - figuring more or less explicitly in some of the aforementioned articles - centers around the notion of event controllability. The key idea is that a negative event outcome reflects unfavorably on the self only if the person has some responsibility for it, which presupposes controllability. For instance, Mark et al. (2003), comparing their own results with those of Tykocinski and colleagues, ar- gued that "it may well be that negative self-relevant outcomes will attenuate hindsight ... only when the person feels some sense of personal responsibility for the outcome (culpability)" (p. 452). Similar arguments can be found in Pezzo (2003, p. 439), Pezzo and Pezzo (2007, pp. 154-157), and Pezzo and Beckstead (2008, p. 493f). Tykocinski and Steinberg (2005), focusing on retroactive pessimism, also discussed the role of controllability and argued that "it is easier to conclude that 'I never had a chance to succeed' when the negative outcomes are uncontrollable" (p. 554). They go on to actually demonstrate that people show more retroactive pessimism in a low-control than in a high-control scenario (Tykocinski \& Steinberg, 2005, Experiment 2). In short, the controllability account of divergent hindsight effects of self-relevant negative events would lead one to expect decreased hindsight bias in high-control situations, where perceived (or claimed; Pezzo \& Pezzo, 2007) unforeseeability serves to reduce responsibility and blame for the outcome. By contrast, it would lead one to expect increased hindsight bias in low-control situations, where retroactive pessimism serves to cope with disappointment.

A second explanation draws on the nature of the hindsight judgments made in the two contradictory bodies of research. As was already mentioned in passing in the description of the studies above, studies on negative decision outcomes asked participants how foreseeable the outcome was, and Tykocinski and colleagues' retroactive pessimism studies used probability judgments as the dependent variable. From the perspective of a recent reconception of hindsight bias (Blank, Nestler, von Collani, \& Fischer, 2008), this is an important difference, since it relates the two sets of studies to two different components of hindsight biasnamely, foreseeability and inevitability ${ }^{1}$ impressions.

Blank et al. (2008) suggested and provided evidence that the phenomenology of the hindsight bias actually encompasses three distinct and empirically separable components: foreseeability and inevitability, as mentioned before, and memory distortion (which is less important in the present context). The foreseeability and inevitability components refer to impressions that event outcomes were (to some degree) foreseeable and inevitable, respectively. Hence, hindsight bias in terms of these components emerges if events are perceived as more foreseeable or inevitable in hindsight than in foresight. Generally, the different hindsight components refer to different entities and serve different psychological functions (among other differences between them; see Blank et al., 2008). Specifically, foreseeability refers to one's subjective state of knowledge at a given point in time, whereas inevitability addresses an objective state of the world.

Clearly, foreseeability is essential in decision outcome studies: What matters is not so much how objectively likely the outcome was but what one could have subjectively known about these real-world contingencies. By contrast, inevitability is central for retroactive pessimism: Consolation can come only from the belief that there was really (i.e., objectively) nothing that could have changed the outcome; whether or not one knew about (i.e., could foresee) these contingencies is largely irrelevant. Hence, 
there is a logical connection between the psychological functions of the components in the present context (i.e., deflection of responsibility vs. reduction of disappointment) and their nature (i.e., referring to a subjective state of mind vs. an objective state of affairs).

In summary, this second explanation of our puzzle maintains that not controllability but "componentness" is the key to understanding the opposite hindsight effects: The foreseeability component is congenial for the effects obtained in decision-making studies, and the inevitability component is the natural vehicle for retroactive pessimism effects. Without this component-to-effect match, the pattern of results obtained in studies on negative self-relevant event outcomes would be less clear-cut. This is not to say that it would disappear altogether, since there might be an independent contribution of controllability, as outlined above. In fact, the two approaches may not so much contradict as supplement each other; in any case, this is in part an empirical question and the topic of the study presented below.

\section{Previous Relevant Evidence for Our \\ Component Explanation}

There are at least two lines of evidence that give the component account some credibility. The first is almost trivial but, nevertheless, important: It is the confirmation that the dependent measures used to assess hindsight bias in the studies introduced above can, in fact, be related to the foreseeability and inevitability components along the lines discussed above. Table 1 gives an overview of the relevant measures and wordings. In three of the decision- making studies that showed a reduction or elimination of hindsight bias, the dependent measures are obvious measures of foreseeability and require no further comment (Louie, 1999; Mark et al., 2003; Mark \& Mellor, 1991). In three others (Louie, 2005; Louie et al., 2000; Pezzo $\&$ Beckstead, 2008), the participants were asked (1) to make hindsight predictions of the outcome or (2) to recall predictions that they had actually made. Despite the difference in design between these two cases (a so-called hypothetical vs. a memory design; ${ }^{2}$ see Pohl, 2007, for an overview of hindsight bias research methodology), both are related to foreseeability: Stating that one (1) would have predicted or (2) in fact did predict the outcome essentially implies that one (claims that one) foresaw this to happen (cf. Fischhoff, 1977, for a similar line of argument). Thus, all the studies that showed reduced hindsight bias for self-relevant negative events essentially captured the foreseeability component of hindsight bias.

By contrast, all of Tykocinski and colleagues' retroactive pessimism studies, as well as those by Sanna and Chang (2003, Experiment 2) and Wann et al. (2008), employed probability ratings. These resemble inevitability judgments, since in both studies an objective state of the world was addressed ("it is highly likely"; "it is inevitable"), as opposed to foreseeability judgments, which address a subjective state of knowledge concerning this state (e.g., "I could not foresee it"). Also, perceiving an outcome as $100 \%$ likely means perceiving it as inevitable. Probability and inevitability are not identical, though, since, for instance, the latter involves an element of causal determina-

Table 1

Measures of Hindsight Bias Used in Studies Involving Self-Relevant Negative Outcomes

Study

Measure/Question Wording

\section{Studies That Showed a Reduction or Elimination of Hindsight Bias}

Louie (1999)

Louie (2005)

Louie, Curren, \& Harich (2000)

Mark, Boburka, Eyssell, Cohen, \& Mellor (2003)

Mark \& Mellor (1991)

Pezzo \& Beckstead (2008)
"I would have predicted that the value would definitely decrease/increase" (direct measure of foreseeability) Hindsight postdictions of outcome (indirectly related to foreseeability)

Recall of foresight likelihood ratings of outcome (indirectly related to foreseeability)

Three-item scale: (1) "I was surprised by the performance of this stock" [reverse coded]; (2) "I could foresee what this stock was going to do"; (3) "It was obvious after the first four-day trading period that this stock was going to perform as it did" (direct measure of foreseeability)

Scaled response alternatives: (1) "I'm not sure I ever saw it coming"; (2) "I wasn't sure, but suspected it was coming"; (3) "I saw it coming all the way" (direct measure of foreseeability)

Recall of foresight likelihood ratings of several outcomes (indirectly related to foreseeability)

Studies That Showed an Increase in Hindsight Bias

Sanna \& Chang (2003)

Experiment 1: inevitability ratings - "what would you have estimated the inevitability that the overall outcome would ultimately turn out to be a failure?" (direct measure of inevitability)

Experiment 2: probability ratings - "what would you have thought the probability of performing poorly would be?" (closely related to inevitability)

Tykocinski (2001) Probability ratings; Experiment 1—of "your chances to get to the store in time"; Experiment 2 - of the chances of winning the election (closely related to inevitability)

Tykocinski, Pick, \& Kedmi Probability ratings; Experiment 1 - of the chances to win the game; Experiment 2 and very similar in Experi(2002) ment 3 - of "your chances of getting to the stipend office and submitting the forms in time" (closely related to inevitability)

Tykocinski \& Steinberg (2005) Probability ratings; Experiment 1 - of their chances of making it to the flight on time considering all that had happened; Experiment 2 - of their chances of reaching the university's grant office before it had closed considering all that had happened (closely related to inevitability)

Wann, Grieve, Waddill, \& Probability ratings of the chances to win the game; replicating Tykocinski et al.'s (2002) method (closely Martin (2008) related to inevitability)

Note-Original wordings (where available) are given in quotation marks. See the text for a detailed discussion of the italicized remarks in parentheses. 
tion, whereas probability applies also to chance events. Hence, they should be substantially but not perfectly related, which, in fact, they turn out to be $(r=.34$ in Blank et al., 2008, Study 4; and $r=.54$ in Nestler, Blank, \& von Collani, 2008, Experiment 2). In addition to these studies using probability measures, one study actually employed a single-item measure of inevitability (Sanna \& Chang, 2003). Overall, then, the evidence summarized in Table 1 clearly supports our idea that the opposing findings in the two sets of studies are related to the difference between foreseeability and inevitability.

More direct evidence has come from a recent study by Blank and Nestler (2006) that investigated local citizens' hindsight cognitions about the city of Leipzig's failed application to host the 2012 Olympic games. The International Olympic Committee's decision in May 2004 to drop Leipzig from the shortlist of applicant cities was a highly negative and self-relevant outcome for the overwhelming majority of participants, since support for the application had been immense in the populace. Interestingly, our study revealed an inverse hindsight bias in terms of foreseeability, resembling the effects obtained in studies involving negative decision outcomes, whereas a strong positive hindsight effect emerged for inevitability impressions, just as in the retroactive pessimism studies. Moreover, reinforcing our argument above, there was correlative evidence to link these effects to different psychological functions. Decreases in foreseeability were stronger in individuals who had shown more commitment to the application (e.g., active support), highlighting the same avoidance of self-blame as in the decision-making studies discussed above; it would have seemed foolish to claim in hindsight that the case one had so eagerly supported had been foreseeably doomed. In contrast, increases in perceived inevitability were strongly and positively related to the intensity of disappointment by the outcome, in line with Tykocinski's (2001) retroactive pessimism idea.

Importantly, returning to the focus of this article, these opposite foreseeability and inevitability shifts were observed in a situation in which there was no personal control whatsoever over the outcome. This suggests that the opposite effects observed in previous studies were probably driven by the different hindsight components, rather than by any differences in the controllability of the event outcomes in question. Supporting this interpretation, Pezzo and Beckstead (2008) found no effect of event controllability on their foreseeability-related measure of hindsight bias.

\section{The Present Research}

Nevertheless, the evidence discussed in favor of this idea is circumstantial, and it would be more convincing to contrast the impact of controllability and hindsight component within one and the same setting. This was the purpose of the present study. Using a scenario approach, we investigated both variables in a fully crossed, withinparticipants design. Note that assessing both foreseeability and inevitability within participants invites consistency effects, since these two often go hand in hand-often, foreseeability presupposes some degree of determined- ness or inevitability - and might, under certain circumstances, even be perceived as synonymous. If so, this restricts the possibility of obtaining opposite hindsight effects for these components, and therefore, the present within-participants investigation must be considered a hard test of our components idea.

In general, with both types of influence having sufficient theoretical and empirical plausibility, we expected that both controllability and hindsight component would affect the level of hindsight bias, although we did not have any prior views about the respective strength of these effects. That is, we expected high controllability and foreseeability to be the ideal combination for reduced or even reversed hindsight bias and low controllability and inevitability to be the ideal combination for enhanced hindsight bias, but we were not so sure about the relative position of the remaining combinations.

\section{METHOD}

\section{Overview}

We used a scenario approach to investigate the effects of the two variables of primary interest, controllability and the hindsight component. Three between-participants experimental conditions were set up in order to establish (1) the amount of hindsight bias and (2) the degree to which the obtained effects were dependent on processes related to the self. In the actor-hindsight condition, the participants read the scenarios, including their outcomes, from the perspective of the actor and provided hindsight foreseeability and inevitability judgments. The participants in the actor-foresight condition read the same scenarios, but without outcome information; this condition provided the necessary comparison to assess the degree of hindsight bias obtained in the actor-hindsight condition. Finally, the participants in the observer-hindsight condition read the same scenarios (with outcome information), but from an outside perspective-hence, lacking self-relevance. This condition served as a motivationally neutral comparison standard for the hindsight perceptions obtained in the actor-hindsight condition, in order to establish the degree to which the latter were influenced by any of the aforementioned self-related motivational processes. Three scenarios were used that had been selected from a larger sample and met certain criteria of suitability, on the basis of the results of a pretest.

\section{Participants}

A total of 210 nonpsychology students (mostly students of law, business, or education; $63 \%$ female; mean age $=22.1$ years, $S D=$ 2.5 years, median $=22$ years) were approached on the university campus or responded to flyers and posters. They received $€ 5$ for half an hour of participation and were randomly assigned to the experimental conditions.

\section{Scenarios}

To ensure generalizability of the results, we used three different scenarios, partly self-generated and partly inspired by similar scenarios employed by Tykocinski and colleagues and Zeelenberg et al. (1998). This also allowed us to manipulate controllability within participants, which would have been impossible if just one scenario had been used. Thus, each participant received the following three scenarios (although in different versions, according to experimental conditions): (1) The eBay scenario was about a person wanting to purchase a Swatch clock at an eBay auction but who is outbid either because the person is quite sure that nobody will make a higher bid and therefore uses the last minutes of the auction to have a cup of coffee in the kitchen (high controllability) or because the Internet connection breaks down and the person does not succeed in reinstalling it within the last minutes of the auction (low controllability). (2) The 
laptop scenario was very similar to the scenario used by Tykocinski (2001, Experiment 1): A person hears that there is a discount offer on a laptop he or she has intended to buy for quite some time. However, the special offer ends this very day, and there is not much time left to rush to the shop. In the end, the shop is already closed upon the person's arrival. In the high-controllability condition, the person thinks too late about getting cash and, therefore, has to take a detour to an ATM; later, the person ignores a road work sign, which leads to a punctured bicycle tire. In the low-controllability condition, the delay is due to rush-hour traffic, a broken-down ATM, and a punctured tire caused by a nail in the street that was hard to spot. (3) The exam scenario involved a student who fails an important final exam, either because the student spent the revision time partying and on a lastminute vacation in the Caribbean (high controllability) or because the student contracted a heavy cold and his or her grandmother died during revision time (low controllability).

The three scenarios had been selected from an initial sample of 12 scenarios on the basis of a pretest $(N=64)$ in which ratings of four different versions of each scenario were obtained (by a fourth of the participants each): high and low controllability, crossed with perspective (actor hindsight vs. observer hindsight; pretesting the actor-foresight version was not deemed necessary). The pretest sought to establish that the scenarios were (1) sufficiently negative and (2) unsurprising (since surprise affects hindsight bias in ways unrelated to the focus of our experiment; see Müller \& Stahlberg, 2007, for a review). Moreover, (3) the participants should be able to identify themselves with the actor in the actor condition or should perceive the scenario as realistic in the observer condition. Finally, (4) there should be a clear difference in perceived controllability between the high- and low-controllability versions of a scenario. All ratings were given on scales from 1 to 9 . The three chosen scenarios had average ratings of 7.0 for negativity ( $9=$ high negativity), 2.7 for surprisingness $(1=$ low surprise $), 6.8$ for identification $(9=$ high identification), and 7.3 for realism $(9=$ high realism $)$. They also differed clearly in terms of perceived controllability, with average ratings of 6.9 for high-controllability versions and 3.9 for lowcontrollability versions $(9=$ high controllability).

In all the hindsight conditions, a section entitled "situation description" contained the body of a given scenario, and a section entitled "event outcome" contained a single sentence presenting the outcome. The latter section was omitted in the actor-foresight condition. The second-person singular was used to refer to the actor in the two actor conditions; in the observer condition, the third-person singular was used, and the person was identified by a name. The text of one exemplary scenario (the laptop scenario), in both controllability versions, can be found in the Appendix.

\section{Design}

The experiment followed a 3 (condition: actor-hindsight vs. actor-foresight vs. observer-hindsight) $\times 2$ (controllability: high vs. low) $\times 2$ (component: foreseeability vs. inevitability) design, with repeated measurement on the last two factors and random assignment to the levels of the first factor. Sixty participants were assigned to the actor-foresight and observer-hindsight conditions. Because of its pivotal role in the analyses (see below), the number of participants in the actor-hindsight condition was raised to 90 . Each participant received either two high-controllability scenarios and one low-controllability scenario or one high-controllability and two low-controllability scenarios. Counterbalancing ensured that (1) each scenario was used equally often in the high- and lowcontrollability version and (2) each of the six possible presentation orders of the three scenarios was used equally often.

\section{Procedure}

The participants were tested in groups of 4-32 people. Group size was not confounded with condition or any other variable, since these were randomly determined by the individual booklets the participants received. They were advised to work through their booklets in the given order and not to browse forth or back. This was essential because the participants were not supposed to see the outcomes in advance and were required to provide their ratings without looking at the scenarios again. Initially, the participants were, depending on perspective (actor vs. observer), instructed to put themselves as much as possible in the position of the actor or to imagine being an observer who happens to witness the described situation. They then read the first scenario, answered a number of items related to it (see below), and continued with the second and third scenarios. Finally, they provided demographic information and were given the opportunity to write open comments on the study (only a few participants did so). Reading through the scenarios and answering all items took about $25 \mathrm{~min}$, on average.

\section{Measures $^{3}$}

After each scenario, the participants first answered, on scales from 1 (don't agree at all) to 6 (totally agree), a set of items intended to measure the foreseeability and perceived inevitability of the (anticipated, in the actor-foresight condition) scenario outcome. Since there were two possible, dichotomous outcomes in each scenario (e.g., to pass or fail the exam, to arrive on time or late), two items each probed these outcomes separately, followed by seven unspecific items (four relating to foreseeability and three to inevitability), in the sense that they asked for "the outcome" only, leaving its definition to the participants. After detailed scale analyses, the specific items were dropped from both scales, along with an unspecific item each, resulting in a three-item foreseeability measure and a two-item measure of inevitability, both consisting of outcome-unspecific items, in agreement with similar scales used in Blank and Nestler (2006) and Blank et al. (2008). The three (translated) foreseeability items were (1) "I know all along how this situation will turn out," (2) "From the development of the situation, only one particular outcome can be expected," and (3) "It is difficult to predict how the event will turn out" (reverse coded). ${ }^{4}$ The two inevitability items were (1) "Under the given circumstances the outcome is completely determined" and (2) "Because of the many factors that can influence the outcome of the situation, the outcome is still open" (reverse coded). All the items were worded in the present tense, because they were used in combination with a hypothetical-design instruction (see Pohl, 2007) to ignore the outcome and answer the foreseeability and inevitability items as if they did not know it. This ensured that any obtained hindsight effects could be truly considered biases from a normative point of view, since the instruction rendered the outcome normatively irrelevant for the judgments. The internal consistencies of the scales were satisfactory, with Cronbach's $\alpha$ s of .79 (foreseeability) and .71 (inevitability), and the scales were moderately correlated with each other ( $r=.37$, averaged across conditions and controllability levels).

Five additional items (using the same 6-point response scale) followed in the hindsight conditions, which were included as manipulation checks and for analyses related to the psychological functions of the foreseeability and inevitability hindsight components (see below). They addressed the emotional consequences of controllability and perspective (actor vs. observer) in appreciating the outcomes of the scenarios. Four items tapped regret, responsibility, guilt, and self-blame. Since the responses to these items were highly consistent (Cronbach's $\alpha=.87$ ), they were combined into a single self-blame scale. A fifth, separate item probed for disappointment. The participants were asked to imagine how they themselves would feel about the outcome (in the actor perspective) or how the actor would feel about it (in the observer perspective). Following the arguments in the introduction, self-blame should be higher in the high-controllability than in the low-controllability scenarios. Moreover, insofar as self-defense processes were already at work in the actor-hindsight condition, the level of self-blame should be reduced, as compared with the observer-hindsight condition. In contrast, disappointment would not necessarily be expected to differ as a function of controllability but should be reduced if some coping - in the sense of retroactive pessimism - has taken place in the actor-hindsight condition. 


\section{RESULTS}

All the results reported below are averaged across scenarios whenever, depending on counterbalancing, the high- or low-controllability conditions comprised two scenarios. Missing values and outliers were extremely rare; therefore, all the analyses were performed on the original data set. ${ }^{5}$ All the statistical tests were bidirectional, unless otherwise noted.

\section{Manipulation Checks}

A 2 (controllability: high vs. low) $\times 2$ (condition: actorhindsight vs. observer-hindsight) ANOVA of the selfblame scores revealed clear main effects of controllability $\left[F(1,147)=127.89, p<.001, \eta_{\mathrm{p}}^{2}=.47\right]$ and condition $\left[F(1,147)=11.71, p<.001, \eta_{\mathrm{p}}^{2}=.07\right]$ and no interaction between these factors. The participants reported more combined regret, responsibility, guilt, and self-blame in the high-controllability scenarios $(M=3.91)$ than in the low-controllability scenarios $(M=2.60)$. In addition, they showed less combined self-blame in the actor-hindsight condition $(M=2.99)$ than in the observer-hindsight condition $(M=3.52)$. Both results suggest that our manipulations worked as planned.

The same ANOVA with disappointment as the dependent variable did not reveal any significant main effects or interactions. This was as expected with respect to controllability; the unexpected lack of a condition effect (actorhindsight vs. observer-hindsight) was probably due to disappointment being close to the ceiling $(M=5.37$ on a scale from 1 to 6 ). Overall, we conclude that our manipulations were successful.

\section{Hindsight Effects}

To investigate hindsight bias as a function of controllability and component, we compared the actor-hindsight condition with the actor-foresight condition. Since the individual foreseeability and inevitability values were determined by averaging the item values (after recoding, if necessary), rather than summing them up, they had the same metric. This allowed us to treat the two measures as withinparticipants levels of the same variable. Hence, we analyzed hindsight effects, using a 2 (controllability: high vs. low) $\times 2$ (component: foreseeability vs. inevitability) $\times$ 2 (condition: actor-hindsight vs. actor-foresight) ANOVA approach. The pattern of means is shown in Figure 1. The ANOVA yielded significant main effects of component $\left[F(1,148)=29.28, p<.001, \eta_{\mathrm{p}}^{2}=.17\right]$ and condition $\left[F(1,148)=6.23, p=.01, \eta_{\mathrm{p}}^{2}=.04\right]$, as well as an interaction between component and condition $[F(1,148)=5.01$, $\left.p=.03, \eta_{\mathrm{p}}^{2}=.03\right]$. Neither the main effect nor any interactions involving controllability (including the three-way interaction) were statistically significant. The main effect of component is noteworthy but theoretically less interesting in the present context; it simply reflects the fact that the participants tended to generally perceive the scenario outcomes as more foreseeable than inevitable.

More important is the condition effect, which reflects hindsight bias: The participants perceived the scenario outcomes as more foreseeable or inevitable in hindsight than in foresight. The component $\times$ condition interaction, however, showed this hindsight effect to differ between components. Specifically, the hindsight effect did not approach significance for the foreseeability component, as followed up by $t$ tests, neither in high-controllability scenarios $\left[M_{\text {hindsight }}=2.81, M_{\text {foresight }}=2.62 ; t(148)=1.10\right.$, $p=.28, d=0.18]$ nor in low-controllability scenarios $\left[M_{\text {hindsight }}=2.64, M_{\text {foresight }}=2.47 ; t(148)=0.82, p=.41\right.$, $d=0.14]$. In contrast, significant hindsight bias emerged for the inevitability component, in high-controllability scenarios $\left[M_{\text {hindsight }}=2.56, M_{\text {foresight }}=2.21 ; t(148)=2.10\right.$,

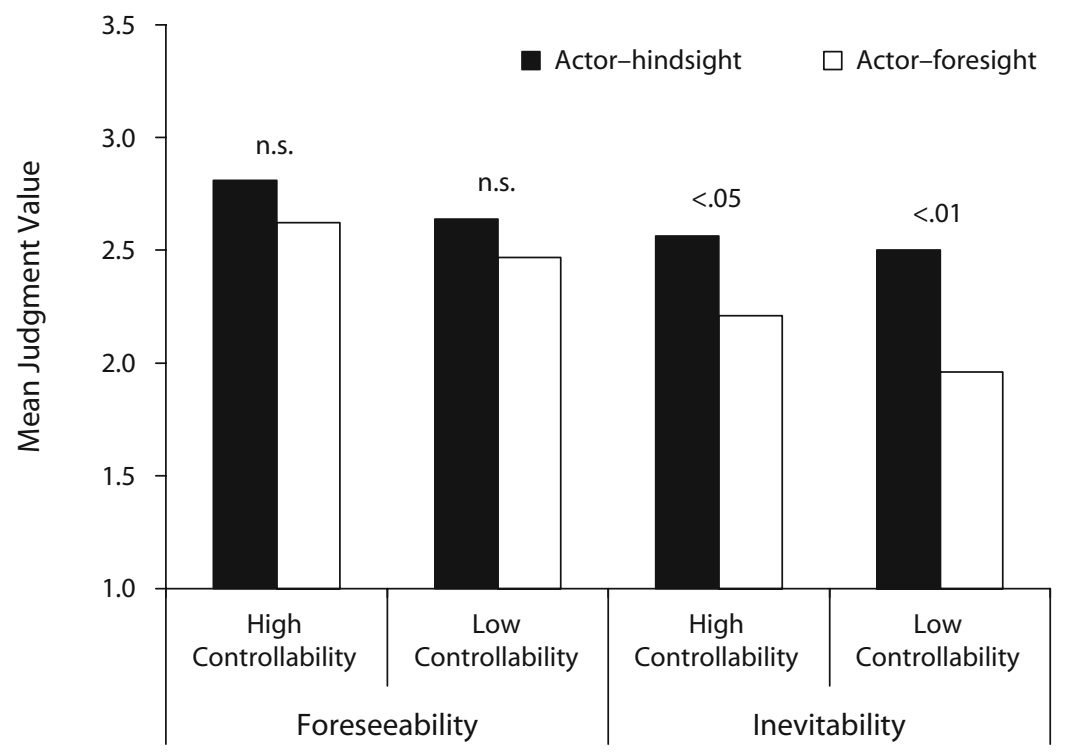

Figure 1. Means of foreseeability and inevitability judgments as a function of controllability and experimental condition. The amount of hindsight bias figures as the difference in height between two adjacent hindsight and foresight bars. 
$p=.04, d=0.35]$ and even more so in low-controllability scenarios $\left[M_{\text {hindsight }}=2.50, M_{\text {foresight }}=1.96 ; t(148)=\right.$ $2.84, p=.005, d=0.48]$. In short, there was significant hindsight bias in terms of inevitability, but not in terms of foreseeability, and moreover, this hindsight bias was strongest in low-controllability scenarios, as was expected (even if this did not lead to statistically significant results involving controllability itself).

The one aspect of these results that did not perfectly meet our expectations was that the lowest amount of hindsight bias was not found for the combination of foreseeability and high controllability but for the foreseeability/low-controllability combination. We are talking here about descriptive differences, though, and given that the amount of hindsight bias was small and statistically nonsignificant in both cases anyway, this should perhaps not be taken too seriously. Apart from this, the most noteworthy nonresult from this analysis of hindsight effects was the nonsignificant influence of controllability. However, we point to subsequent analyses showing that controllability still has a role to play.

\section{Self-Relevance Effects on Hindsight Impressions}

In order to determine whether any of the hindsight (non)effects found in the previous analysis were due to the self-relevance of the outcomes, we compared the actor-hindsight participants' impressions with those of the observer-hindsight participants. Since the latter group was not asked to identify with the actors, the scenario outcomes should be much less self-relevant for them than for the imagined-actor participants. The same ANOVA as above (apart from the different conditions involved) showed a main effect of hindsight component as before $[F(1,148)=$ $\left.40.10, p<.001, \eta_{\mathrm{p}}^{2}=.21\right]$, as well as an interaction between component and condition $[F(1,148)=9.47, p=$ $\left..002, \eta_{\mathrm{p}}^{2}=.06\right]$. No other significant effects emerged.

The pattern of means is presented in Figure 2. The only significant individual comparison is the one for the foreseeability/high-controllability combination $\left[M_{\text {actor }}=\right.$ $\left.2.81, M_{\text {observer }}=3.23 ; t(148)=2.26, p=.03, d=0.38\right]$. That is, the observers found the scenario outcomes more foreseeable than did the imagined-actor participants. The respective difference for the foreseeability/lowcontrollability combination can be considered marginally significant if interpreted unidirectionally $\left[M_{\text {actor }}=2.64\right.$, $M_{\text {observer }}=2.96 ; t(148)=1.45, p=.07$ (one-tailed), $d=$ $0.24]$. The comparisons involving inevitability, however, showed little difference between the imagined-actor participants and observers [inevitability/high-controllability, $M_{\text {actor }}=2.57$ vs. $M_{\text {observer }}=2.64, t(148)=0.47, p=.64$, $d=0.08$; inevitability/low-controllability, $M_{\text {actor }}=2.50$ vs. $M_{\text {observer }}=2.44, t(148)=0.28, p=.78, d=-0.05$ (the minus sign indicating that perceived inevitability was numerically stronger in the actor condition)].

These follow-up analyses show that the lack of hindsight bias in the foreseeability component, as identified in the previous section, seems to be related to the actor perspective. This holds in particular for high-controllability scenarios, whereas a trend that is at least similar can be seen in low-controllability scenarios, too. On the inevitability side, though, there is no evidence that the hindsight effects found above depended on the self-relevance of the outcomes; the fact that the observers showed essentially the same effects suggests that what we have here may just be the ordinary, cold, cognitive hindsight bias that we are already familiar with. Again, we have to point to further analysis to make a better case for motivated hindsight bias in the sense of retroactive pessimism.

\section{Correlational Analyses}

Our last set of analyses sought to provide direct evidence that the hindsight effects obtained are linked, in theoretically meaningful ways, to specific emotional functions. Table 2 presents correlations between self-blame and disappointment, on the one hand, and foreseeability and inevitability

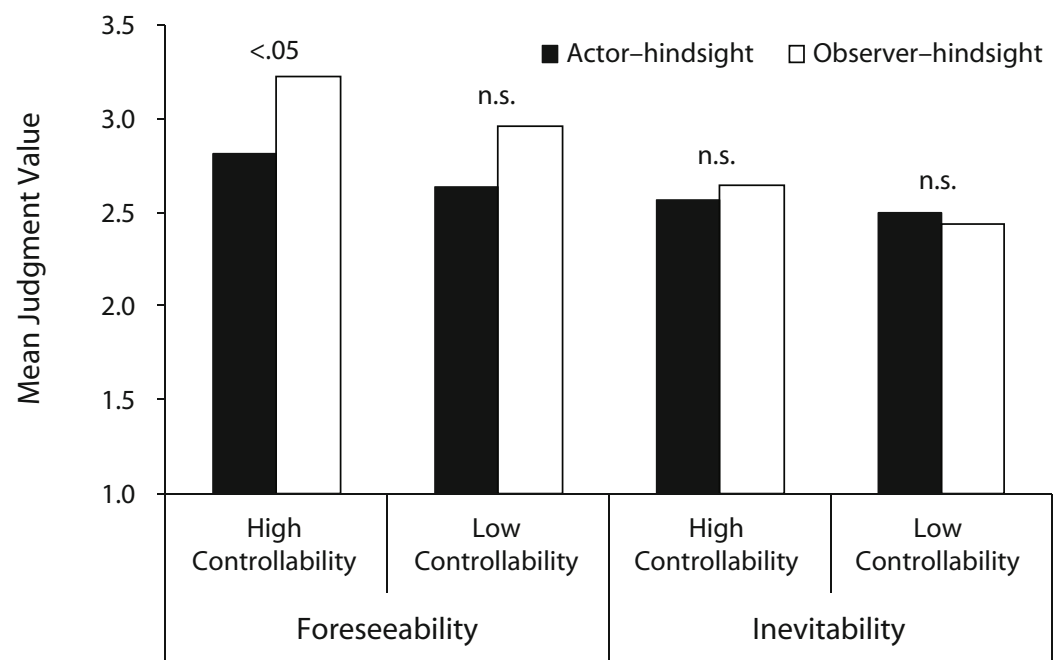

Figure 2. Means of foreseeability and inevitability judgments as a function of controllability and experimental condition. The actor-hindsight bars are the same as those in Figure 1. The height difference between two adjacent actor and observer bars reflects the influence of self-relevance on hindsight impressions. 
Table 2

Correlations Between Emotions and Hindsight Components

\begin{tabular}{lccccc}
\hline & \multicolumn{2}{c}{ Actor-Hindsight } & & \multicolumn{2}{c}{ Observer-Hindsight } \\
\cline { 2 - 3 } \cline { 5 - 6 } Condition/Measure & Self-Blame & Disappointment & & Self-Blame & Disappointment \\
\hline High controllability & & & & & \\
$\quad$ Foreseeability & $+.28^{* *}$ & -.09 & & +.18 & -.01 \\
Inevitability & $+.26^{*}$ & -.13 & & $+.27^{*}$ & -.12 \\
Low controllability & & & & \\
$\quad$ Foreseeability & +.14 & $-.22^{*}$ & & +.15 & +.03 \\
Inevitability & +.13 & $-.32^{* *}$ & & +.05 & -.01 \\
${ }^{*} p<.05 .{ }^{* *} p<.01$. & & & &
\end{tabular}

impressions, on the other, for various combinations of scenario controllability (high vs. low) and hindsight perspective (actor vs. observer). Focusing on the actor-hindsight condition first, we note that the strongest correlation of self-blame is with foreseeability in high-controllability scenarios, whereas the strongest correlation of disappointment is with inevitability in low-controllability scenarios. This is exactly what is to be expected from the self-blame avoidance and from the retroactive pessimism point of view, respectively. Under high controllability, foreseeability is conducive to self-blame: The higher the perceived foreseeability of the negative outcome, the more people have to blame themselves for it - hence, the positive correlation between these variables. Furthermore, under low controllability, perceived inevitability is prototypically suited to reduce disappointment (there was nothing that could be done about it) - hence, the negative correlation between the latter variables. Proceeding from one of these prototypical combinations toward the other, the (absolute) magnitudes of the correlations decrease, indicating the decreasing importance of the respective emotions under those circumstances.

The correlations in the observer-hindsight condition, by comparison, do not show much of a systematic pattern and are generally lower (with the exception of the .27 correlation of self-blame with inevitability in high-control scenarios, for which we have no explanation except a possible Type I error). In short, the observer participants' hindsight impressions did not have much emotional significance for them. In turn, this underscores the motivational nature of the hindsight effects in the actor-hindsight condition, as is predicted by both the self-blame avoidance and retroactive pessimism views and by the idea of motivated hindsight bias in general (e.g., Campbell \& Tesser, 1983; Musch \& Wagner, 2007; Pezzo \& Pezzo, 2007).

Finally, it is important to note that in these analyses, unlike in the others presented so far, the pattern of results follows a grouping according to controllability, rather than according to the hindsight component. Specifically, self-blame played a role in high-controllability scenarios but did not in low-controllability scenarios, practically regardless of component. The opposite controllabilitydriven pattern, although less distinctive, emerges for disappointment. In summary, our correlational analyses established support for a motivational hindsight bias, in terms of self-blame avoidance and retroactive pessimism, and also indicated that controllability may not be so unimportant after all for these effects.

\section{DISCUSSION}

Let us briefly review the three major sets of findings from our study. (1) Hindsight bias followed component: There was significant hindsight bias in terms of inevitability, but not in terms of foreseeability. Descriptively, the amount of inevitability hindsight bias was stronger in low-controllability scenarios. (2) Actor-observer differences in hindsight impressions followed component as well. Specifically, the actors found the scenario outcomes less foreseeable than did the observers; this was a significant difference with high-controllability scenarios and a trend with low-controllability scenarios. Furthermore, the inevitability impressions of the actors and observers were almost identical at both levels of controllability. (3) Correlations between self-blame and disappointment and hindsight impression were in line with both the self-blame avoidance and the retroactive pessimism accounts. Remarkably, these effects followed controllability rather than component (descriptively, though, the disappointmentinevitability correlation was somewhat stronger than the disappointment-foreseeability correlation).

Three conclusions can be derived from these findings. First, for the prototypical combination of high-controllability and foreseeability impressions, there is straightforward supportive evidence from all three analyses for a self-blame avoidance mechanism sensu Mark and Mellor (1991), Louie (1999), and Pezzo and Pezzo (2007). Second, for the prototypical combination of low-controllability and inevitability impressions, there is some support, mostly from the correlational analysis, for retroactive pessimism as suggested by Tykocinski and colleagues (Tykocinski, 2001; Tykocinski et al., 2002; Tykocinski \& Steinberg, 2005). In addition, there is significant hindsight bias, as was expected, and the actor-observer difference, although small and not significant, is at least in the right direction. Hence, the overall picture that emerges from this combined evidence is in line with the idea of retroactive pessimism, although it is not as convincing as in the self-blame avoidance case. In sum, both of the motivational mechanisms highlighted in previous studies on hindsight bias for self-relevant negative event outcomes were operating in our experiment, under their respective prototypical combinations of controllability and hindsight component as identified in the introduction.

Having established this, we can now revisit the main question of this experiment - namely, the question regarding the relative importance of controllability and the hind- 
sight component for the opposite motivational hindsight effects obtained here and in earlier studies. In this respect, the third clear conclusion we can derive from our findings is that the hindsight component matters. In two out of three analyses, component had a clear effect on hindsight impressions, and there was at least some supportive evidence in the third, correlational analysis. Note that this holds in spite of the likely presence of a trend toward consistency between foreseeability and inevitability judgments (as inferred from the moderate correlation of $r=.37$ between the two components), which would work against observing any differences between the components, and this, in turn, makes our conclusion even stronger. Hence, as would be expected from our theoretical analysis and from an earlier study (Blank \& Nestler, 2006), the opposite motivational effects are logically tied to the nature and functions of the different hindsight components. By implication, this also supports the underlying idea of fundamentally different hindsight components (Blank et al., 2008).

What about the role of controllability, though? The evidence is mixed. Statistically, there was little support from the hindsight bias and self-relevance analyses. Note, however, that the inevitability hindsight bias was descriptively stronger in low-controllability scenarios (see Figure 1). Also, the motivated reduction of foreseeability, as shown by the actor-hindsight participants, as compared with the observer-hindsight participants, was descriptively larger in high-controllability scenarios (see Figure 2). These trends might reflect a genuine but small effect of controllability that went undetected for power reasons. This interpretation is reinforced by the results of the correlational analysis, which did follow a controllability pattern. Hence, with tentative support from two analyses and stronger support from a third, it seems fair to say that a contribution of controllability to our opposite motivational hindsight effects remains a viable option.

Even so, we note that in our study, the hindsight component influenced our participants' hindsight perceptions more strongly than controllability did. This is a remarkable finding, given that controllability has so far been the only solution offered for the persistent anomaly regarding hindsight effects of self-relevant negative events. With our study, a strong alternative candidate emerges, and we hope that future theorizing and research will clarify the conditions under which the hindsight component or controllability is more important in terms of generating self-blame avoidance or retroactive pessimism effects.

\section{AUTHOR NOTE}

This study was supported by the German Research Foundation (BL 493/2-1). We thank Aileen Oeberst and Sascha Krause for their assistance in data collection and Steffen Nestler, as well as two anonymous reviewers, for helpful comments on an earlier version of this article. Correspondence concerning this article should be addressed to H. Blank, Department of Psychology, University of Portsmouth, King Henry Building, King Henry I Street, Portsmouth PO1 2DY, England (e-mail: hartmut.blank@port.ac.uk).

\section{REFERENCES}

BLANK,H.,FISCHER, V., \&ERDFELDER, E. (2003). Hindsightbias in political elections. Memory, 11, 491-504. doi:10.1080/09658210244000513

Blank, H., Musch, J., \& Pohl, R. F. (2007). Hindsight bias: On being wise after the event. Social Cognition, 25, 1-9. doi:10.1521/ soco.2007.25.1.1

Blank, H., \& Nestler, S. (2006). Perceiving events as both inevitable and unforeseeable in hindsight: The Leipzig candidacy for the Olympics. British Journal of Social Psychology, 45, 149-160. doi:10.1348/014466605X52326

Blank, H., Nestler, S., von Collani, G., \& Fischer, V. (2008). How many hindsight biases are there? Cognition, 106, 1408-1440. doi:10.1016/j.cognition.2007.07.007

CAmpbell, J. D., \& Tesser, A. (1983). Motivational interpretations of hindsight bias: An individual difference analysis. Journal of Personality, 51, 605-620. doi:10.1111/j.1467-6494.1983.tb00868.x

ERdFelder, E., \& Buchner, A. (1998). Decomposing the hindsight bias: A multinomial processing tree model for separating recollection and reconstruction in hindsight. Journal of Experimental Psychology: Learning, Memory, \& Cognition, 24, 387-414. doi:10.1037/0278 $-7393.24 .2 .387$

FischноғF, B. (1975). Hindsight $\neq$ foresight: The effect of outcome knowledge on judgment under uncertainty. Journal of Experimental Psychology: Human Perception \& Performance, 1, 288-299. doi:10.1037/0096-1523.1.3.288

FischHoff, B. (1977). Perceived informativeness of facts. Journal of Experimental Psychology: Human Perception \& Performance, 3, 349358. doi:10.1037/0096-1523.3.2.349

Hawkins, S. A., \& Hastie, R. (1990). Hindsight: Biased judgments of past events after the outcomes are known. Psychological Bulletin, 107, 311-327. doi:10.1037/0033-2909.107.3.311

Hell, W., Gigerenzer, G., Gauggel, S., Mall, M., \& Müller, M. (1988). Hindsight bias: An interaction of automatic and motivational factors? Memory \& Cognition, 16, 533-538.

Hoffrage, U., \& Pohl, R. (2003). Research on hindsight bias: A rich past, a productive present, and a challenging future. Memory, 11, 329335. doi:10.1080/09658210344000080

Louie, T. A. (1999). Decision makers' hindsight bias after receiving favorable and unfavorable feedback. Journal of Applied Psychology, 84, 29-41. doi:10.1037/0021-9010.84.1.29

Louie, T. A. (2005). Hindsight bias and outcome-consistent thoughts when observing and making service provider decisions. Organizational Behavior \& Human Decision Processes, 98, 88-95. doi:10.1016/j .obhdp.2005.03.004

Louie, T. A., Curren, M. T., \& Harich, K. R. (2000). "I knew we would win": Hindsight bias for favorable and unfavorable team decision outcomes. Journal of Applied Psychology, 85, 264-272. doi:10 $.1037 / 0021-9010.85 .2 .264$

Mark, M. M., Boburka, R. R., Eyssell, K. M., Cohen, L. L., \& MelLOR, S. (2003). "I couldn't have seen it coming": The impact of negative self-relevant outcomes on retrospections about foreseeability. Memory, 11, 443-454. doi:10.1080/09658210244000522

MARK, M. M., \& MelLor, S. (1991). Effect of self-relevance of an event on hindsight bias: The foreseeability of a layoff. Journal of Applied Psychology, 76, 569-577. doi:10.1037/0021-9010.76.4.569

Müller, P. A., \& STAhlberg, D. (2007). The role of surprise in hindsight bias: A metacognitive model of reduced and reversed hindsight bias. Social Cognition, 25, 165-184. doi:10.1521/soco.2007.25.1.165

Musch, J., \& WAGNer, T. (2007). Did everybody know it all along? A review of individual differences in hindsight bias. Social Cognition, 25, 64-82. doi:10.1521/soco.2007.25.1.64

Nestler, S., Blank, H., \& von Collani, G. (2008). Hindsight bias doesn't always come easy: Causal models, cognitive effort, and creeping determinism. Journal of Experimental Psychology: Learning, Memory, \& Cognition, 34, 1043-1054. doi:10.1037/0278-7393.34.5.1043

Pezzo, M. V. (2003). Surprise, defence, or making sense: What removes hindsight bias? Memory, 11, 421-441. doi:10.1080/ 09658210244000603

Pezzo, M. V., \& Beckstead, J. W. (2008). The effects of disappointment on hindsight bias for real-world outcomes. Applied Cognitive Psychology, 22, 491-506. doi:10.1002/acp.1377

Pezzo, M. V., \& Pezzo, S. P. (2007). Making sense of failure: A motivated model of hindsight bias. Social Cognition, 25, 147-164. doi:10 $.1521 /$ soco.2007.25.1.147

PoHL, R. F. (ED.) (2004). Cognitive illusions: A handbook on fallacies and biases in thinking, judgement and memory. Hove, U.K.: Psychology Press. 
PoHL, R. F. (2007). Ways to assess hindsight bias. Social Cognition, 25, 14-31. doi:10.1521/soco.2007.25.1.14

Pohl, R. F., Eisenhauer, M., \& Hardt, O. (2003). SARA: A cognitive process model to simulate the anchoring effect and hindsight bias. Memory, 11, 337-356. doi:10.1080/09658210244000487

RENNER, B. (2003). Hindsight bias after receiving self-relevant health risk information: A motivational perspective. Memory, 11, 455-472. doi:10.1080/09658210244000531

Roese, N. J., \& Olson, J. M. (1996). Counterfactuals, causal attributions, and the hindsight bias: A conceptual integration. Journal of Experimental Social Psychology, 32, 197-227. doi:10.1006/jesp.1996.0010

Sanna, L. J., \& Chang, E. C. (2003). The past is not what it used to be: Optimists' use of retroactive pessimism to diminish the sting of failure. Journal of Research in Personality, 37, 388-404. doi:10.1016/ S0092-6566(03)00013-8

Sedikides, C., \& Gregg, A. P. (2008). Self-enhancement: Food for thought. Perspectives on Psychological Science, 3, 102-116. doi:10 $.1111 / \mathrm{j} .1745-6916.2008 .00068 . \mathrm{x}$

StAhlbERG, D., \& MAass, A. (1998). Hindsight bias: Impaired memory or biased reconstruction? In W. Stroebe \& M. Hewstone (Eds.), European review of social psychology (Vol. 8, pp. 105-132). Chichester, U.K.: Wiley.

TYкосINSKI, O. E. (2001). I never had a chance: Using hindsight tactics to mitigate disappointments. Personality \& Social Psychology Bulletin, 27, 376-382. doi:10.1177/0146167201273011

Tykocinski, O. E., PIck, D., \& Kedmi, D. (2002). Retroactive pessimism: A different kind of hindsight bias. European Journal of Social Psychology, 32, 577-588. doi:10.1002/ejsp.120

Tykocinski, O. E., \& Steinberg, N. (2005). Coping with disappointing outcomes: Retroactive pessimism and motivated inhibition of counterfactuals. Journal of Experimental Social Psychology, 41, 551-558. doi:10.1016/j.jesp.2004.12.001

Wann, D. L., Grieve, F. G., Waddill, P. J., \& Martin, J. (2008). Use of retroactive pessimism as a method of coping with identity threat: The impact of group identification. Group Processes \& Intergroup Relations, 11, 439-450. doi:10.1177/1368430208095399

Wasserman, D., Lempert, R. O., \& Hastie, R. (1991). Hindsight and causality. Personality \& Social Psychology Bulletin, 17, 30-35. doi:10.1177/0146167291171005
Zeelenberg, M., van Dijk, W. W., van der Pligt, J., Manstead, A. S. R., VAN EMPELEN, P., \& REINDERMAN, D. (1998). Emotional reactions to the outcomes of decisions: The role of counterfactual thoughts in the experience of regret and disappointment. Organizational Behavior \& Human Decision Processes, 75, 117-141. doi:10.1006/obhd.1998.2784

\section{NOTES}

1. In previous publications (e.g., Blank \& Nestler, 2006; Blank et al., 2008), we have used either of the terms inevitability or necessity to refer to this hindsight component. In this article, we will use inevitability throughout.

2. The key difference between hypothetical and memory designs is that in the former, participants' hindsight judgments (i.e., with outcome knowledge) are compared with other participants' foresight judgments (i.e., without outcome knowledge), whereas in a memory design, their recollected (after outcome feedback) foresight judgments are compared with their actual foresight judgments made earlier. The present study uses a hypothetical design.

3 . In addition to the measures reported in the main text, we also employed some personality scales and items probing for commitment to and realism of the scenarios. Since these measures were not meaningfully related to our main results, we do not discuss them in detail.

4. Readers may note that the first foreseeability item differs from the other items in that it is worded in the first-person singular (i.e., reflects what may be called personal foreseeability), whereas the two remaining items use the passive voice (hence reflecting general foreseeability). From the scale analysis, it is clear that this did not affect their functioning within the foreseeability scale. Moreover, we reran all of our analyses presented in the Results section, using only the first item as a measure of foreseeability. Although being statistically weaker overall (as a result of the lower reliability of a single item), the pattern of results in all of the analyses was essentially the same. Hence, our findings and conclusions do not depend on particularities of the foreseeability scale.

5. Control analyses with outliers ( $>3 S D$ s from the mean) eliminated yielded essentially the same results; none of our conclusions would have to be changed as a function of including or excluding outliers.

\section{APPENDIX \\ Laptop Scenario (Actor Conditions)}

\section{Situation Description (High-Controllability Version)}

For quite some time you have been thinking about getting a new laptop. However, the model you were focusing on has been too expensive as yet. This afternoon you meet a friend who tells you that he bought this laptop yesterday and is quite enthusiastic about it. Two days ago, a new computer store was opened out of town. As an introductory offer, this very laptop had been reduced by $€ 350$. The price has to be paid in cash though, and the offer is valid only until tonight. After that, the price will increase again.

It is already quite late. You wonder if you can make it there before closing time. After all, the store is out of town and you'd have to go right through the city centre. You get started as the laptop is very important for you. Reckoning that you'd be too slow on a bus during rush hour, you go home to get your bike. On your bike, you ride quickly to the store.

On the way you remember that you need cash, so you turn around and go to an ATM you know. Remembering earlier would have saved you this detour. [Low-controllability version: On the way you stop at an ATM to get some cash. Unfortunately, the ATM doesn't work. You therefore go to another ATM even though this involves a detour.]

Some two kilometers before the store you notice a road work sign. In order to get on faster, you decide to ignore the warning. This is bad news however, as it leads to your front tire being punctured. [Low-controllability version: Some two kilometers before the store you notice that there's something wrong with your front tire. You check - a nail that must have been on the bike path sticks in your front tire, which is now flat.] You lock the bike to a fence and proceed on foot. Minutes later you arrive at the store.

\section{Event Outcome}

The store is already closed-you are late. 